\title{
Construction of orthotopic xenograft mouse models for human pancreatic cancer
}

\author{
LEI DAI ${ }^{1,2}$, CAIDE LU $^{1,2}, \mathrm{XI} \mathrm{YU}^{2}$, LONG-JUN DAI ${ }^{3}$ and JEFF X. $\mathrm{ZHOU}^{1}$ \\ ${ }^{1}$ Department of Pathology, Ningbo University School of Medicine, Ningbo, Zhejiang 315211; \\ ${ }^{2}$ Department of Hepatobiliary and Pancreatic Surgery, Lihuili Hospital, Ningbo, Zhejiang 315040, P.R. China; \\ ${ }^{3}$ Department of Surgery, Vancouver General Hospital, University of British Columbia, Vancouver, BC V5Z IL8, Canada
}

Received July 22, 2014; Accepted June 1, 2015

DOI: $10.3892 / \mathrm{etm} .2015 .2642$

\begin{abstract}
Animal models are indispensable for the study of tumorigenesis and the development of anti-cancer drugs for human pancreatic cancer. In the present study, two orthotopic xenograft mouse models were developed. AsPC-1 human pancreatic cancer cells were stably labeled with red fluorescent protein (RFP) and injected subcutaneously into nude mice. For the orthotopic tumor mass model, the formed subcutaneous tumors were cut into blocks and implanted into the pancreas of nude mice via laparotomy. For the Matrigel $^{\mathrm{TM}}$ tumor block model, solidified Matrigel containing RFP-labeled AsPC-1 cells was cut into blocks and implanted into the pancreas of nude mice. A subcutaneous tumor xenograft model was used as a control. Tumor growth and metastasis were assessed using an in vivo fluorescence imaging system. Thirty-six days after implantation, all mice from the two orthotopic xenograft models ( $\mathrm{n}=20$ per group) and $55 \%$ of the subcutaneous xenograft mice $(n=20)$ developed tumors. The tumor growth rate was significantly higher in the orthotopic models than that in the subcutaneous model $(\mathrm{P}<0.01)$. Metastasis to organs such as the liver was observed in the orthotopic tumor models. Histological examination showed that the tumors were poorly differentiated adenocarcinomas. In conclusion, two orthotopic xenograft mouse models of human pancreatic cancer were established; these exhibited greater tumor growth and metastasis than the subcutaneous xenograft mouse model.
\end{abstract}

Correspondence to: Dr Caide Lu, Department of Hepatobiliary and Pancreatic Surgery, Ningbo Lihuili Hospital, 58 Xingning Road, Ningbo, Zhejiang 315040, P.R. China

E-mail: lucaide@nbu.edu.cn

Dr Jeff X. Zhou, Department of Pathology, Ningbo University School of Medicine, 818 Fenghua Road, Ningbo, Zhejiang 315211, P.R. China

E-mail: zhouxiwu@nbu.edu.cn

Key words: pancreatic carcinoma, xenograft tumor model, orthotopic tumor model

\section{Introduction}

Pancreatic cancer is one of the most fatal malignances, with a poor overall 5-year survival rate of $<5 \%$ (1-3). In most patients, the tumors already have local or distal metastasis at diagnosis and therefore are unresectable. The tumorigenesis and metastasis of pancreatic cancer have been extensively studied (4), with most studies being performed in vitro using tumor cell lines. Although tumor cell lines represent a useful model for studying the biochemical and molecular changes of this malignancy, they lack an orthotopic environment, which is crucial for analyses of tumorigenesis, metastasis and response to treatments. In vivo animal models represent a more desirable approach for the study of this malignancy and cancer diseases as a whole.

A number of in vivo animal models have been used to study pancreatic cancer. The most classical model is the subcutaneous injection of human tumor cells into an immunocompromised mouse, such as the severely compromised immunodeficient mouse (5). This model has certain advantages, including the simplicity of the procedure, its less invasive nature and the ease of observations of tumor growth and response to treatment; however, it still lacks an orthotopic environment for pancreatic tumor formation. As an improvement of the subcutaneous injection, the orthotopic injection of tumor cells into the pancreas of the mouse produces a xenograft model, which mimics the environment for cancer cells to grow and migrate; however, the cell injection method can generate certain problems, such as the leakage of cells into surrounding tissues. An alternative method to the orthotopic cell injection model is to mix tumor cells with Matrigel ${ }^{\mathrm{TM}}$ before the orthotopic injection (6). Matrigel is a mixture of extracellular matrix proteins secreted by mouse sarcoma cells and has been used extensively for in vitro cell culture due to its resemblance to the complex extracellular environment found in numerous tissues $(7,8)$. Mixing tumor cells with Matrigel could potentially reduce the leakage of tumor cells.

In order to establish appropriate mouse xenograft models for the study of tumorigenesis and evaluations of novel therapeutics for pancreatic cancer, two orthotopic xenograft mouse models were developed in the present study by directly implanting a tumor mass or Matrigel-tumor cell block into the pancreas of a nude mouse. The results were analyzed. 


\section{Materials and methods}

Preparation of pancreatic cells stably expressing red fluorescent protein (RFP). AsPC-1 human pancreatic cancer cells were purchased from the Cell Bank of the Chinese Academy of Sciences (Wuhan, China). AsPC-1 cells were cultured in RPMI-1640 medium (Hyclone Laboratories, Inc., Logan, UT, USA) containing $10 \%$ heat-inactivated fetal bovine serum (FBS) (Hyclone Laboratories, Inc.), penicillin (100 U/ml) and streptomycin (100 U/ml). 293T cells (The Cell Bank of the Chinese Academy of Sciences, Wuhan, China) used for producing lentiviral particles were cultured in Dulbecco's modified Eagle's medium (Hyclone Laboratories, Inc.) containing 10\% heat-inactivated FBS, penicillin $(100 \mathrm{U} / \mathrm{ml})$ and streptomycin $(100 \mathrm{U} / \mathrm{ml})$. All cells were cultured in a humidified incubator at $37^{\circ} \mathrm{C}$ with $5 \% \mathrm{CO}_{2}$ in the atmosphere. A lentiviral system (pLenti-DsRed-Monomer) expressing RFP was purchased from Shanghai Invitrogen Biotechnology Co., Ltd. (Shanghai, China). AsPC-1 cells in the logarithmic growth phase were trypsinized and seeded into six-well plates at $4.5 \times 10^{5}$ cells/well. The RFP-expressing lentiviral vectors were added to the cells slowly. After $48 \mathrm{~h}$, the expression of RFP was detected using fluorescence microscopy. The cells with the highest levels of RFP expression were chosen for continued culture in a medium containing antibiotic Blasticidin $(0.3 \mu \mathrm{g} / \mathrm{ml}$; Shanghai Invitrogen Biotechnology Co., Ltd.) for the selection of RFP-positive cells. Selected cells were referred to as AsPC-1-dsRed cells and maintained in culture in the presence of Blasticidin $(0.2 \mu \mathrm{g} / \mathrm{ml})$.

Preparation of animal models. BALB/C (nu/nu) nude mice of both genders aged 4-6 weeks and weighing 16-22 $\mathrm{g}$ were purchased from Shanghai SLAC Laboratory Animal Co, Ltd. (Shanghai, China). The mice were housed in a pathogen-free environment at a temperature of $25^{\circ} \mathrm{C}$ and relative air humidity between 45 and $50 \%$. All surgeries were performed in a sterile environment. Sixty mice were randomly divided into three groups of 20: Orthotopic tumor mass, orthotopic Matrigel-cell block and subcutaneous tumor cell injection.

In order to establish an orthotopic tumor block xenograft model, subcutaneous xenograft tumors were generated by injecting AsPC-1-dsRed cells subcutaneously into the dorsal flank of the mice. When the tumors grew to a size of $\sim 1 \mathrm{~cm}^{3}$, the mice were sacrificed by cervical dislocation and the tumors were isolated and cut into $40-\mathrm{mm}^{3}$ blocks. The mice $(\mathrm{n}=20)$ were anesthetized by an intraperitoneal injection of $2 \%$ sodium pentobarbital solution. A 1-cm longitudinal skin incision was made on the left upper axillary region of the abdomen of the mouse, the peritoneum was opened and the pancreas was well exposed. The pancreatic capsule was cut open and a piece of $40-\mathrm{mm}^{3}$ tumor block was implanted into the pancreas using the purse-string suture surgical method with an 8-0 absorbable suture. The pancreas was put back into the abdominal cavity gently and the surgical opening was closed using a 6-0 absorbable surgical suture (9).

In order to establish an orthotopic Matrigel block xenograft model, AsPC-1-dsRed cells in single suspension were prepared and mixed with Matrigel (BD Biosciences, Bedford, MA, USA) at a ratio of $2.5 \times 10^{7}$ cells $/ \mathrm{ml}$ Matrigel to form a $1-\mathrm{cm}^{3}$ Matrigel-tumor cell block at room temperature. Following solidification, the block was cut into smaller blocks of $\sim 40 \mathrm{~mm}^{3}$ each. The mice $(\mathrm{n}=20)$ were anesthetized and operated on in the same way as those for the orthotopic tumor block xenograft model. Following the implantation of the Matrigel block, the pancreas was put back into the abdominal cavity gently and the surgical opening was closed.

In order to establish a subcutaneous xenograft model, an AsPC-1-dsRed single cell suspension was prepared at a concentration of $5 \times 10^{7}$ cells $/ \mathrm{ml}$. Cells $\left(1 \times 10^{7}\right.$ cells in $0.2 \mathrm{ml}$ culture medium) were injected subcutaneously in the dorsal flank region of mice $(n=20)$. The animal use protocol was approved by the Animal Care Committee of Ningbo University (Ningbo, China).

Histological examination. The tumor size in the subcutaneous xenograft model was measured every 6 days using a vernier caliper, while in the two orthotopic xenograft models the size of the tumor was measured using an in vivo animal fluorescence imager (Carestream Health, Rochester, NY, USA) (excitation wavelength, $530 \mathrm{~nm}$; emission wavelength, $600 \mathrm{~nm}$ ). The average tumor volume $(\mathrm{V})$ was calculated using the following equation: $\mathrm{V}=\mathrm{A} \times \mathrm{B}^{2} \times 0.5$ (A, long diameter; $\mathrm{B}$, short diameter) (10). The tumor growth rate (U) was calculated using the equation $\mathrm{U}=\mathrm{V}\left(\mathrm{mm}^{3}\right) /$ tumor-bearing time (days). Tumor metastasis was also monitored using the in vivo animal fluorescence imager (Carestream Health). Ten weeks after implantation, the mice were sacrificed and dissected. The tumor volume was measured and the invasion and metastasis were examined. The primary and metastatic tumors were collected, fixed in $10 \%$ neutral formalin and embedded in paraffin. Serial sections of $4-\mu \mathrm{m}$ thickness were cut for hematoxylin and eosin staining.

Western blot analysis. Cultured cells were lysed using radioimunoprecipitation assay buffer containing $50 \mathrm{mM}$ Tris- $\mathrm{HCl}$ (pH 7.4), $150 \mathrm{mM} \mathrm{NaCl}, 1 \% \mathrm{NP}-40$ and $0.25 \%$ sodium deoxycholate, plus $1 \mathrm{mM}$ phenylmethylsulfonyl fluoride and $1 \mathrm{X}$ Roche cOmplete Mini Protease Inhibitor (Roche Diagnostics Corporation, Indianapolis, IN, USA). Protein samples were loaded onto a $12 \%$ SDS-PAGE, and run at a constant current. Following electrophoresis, proteins were transferred to a nitrocellulose membrane. The membrane was blocked with 4\% fat-free milk powder in phosphate-buffered saline and incubated with rabbit polyclonal anti-RFP antibody (R10367; Life Technologies, Grand Island, NY, USA) and subsequently with anti-rabbit IgG secondary antibody (1:5,000; sc-2004; Santa Cruz Biotechnology, Inc. CA, USA). Protein signals were detected using enhanced chemiluminescence reagents (Pierce Biotechnology, Inc., Rockford, IL, USA).

Statistical analysis. SPSS software, version 18.0 (SPSS, Inc., Chicago, IL, USA) was used to perform statistical analysis. Tumor volumes and average growth rates are expressed as the mean \pm standard deviation. Analysis of variance was used to detect any statistically significant differences in the tumor volumes and growth rates among the three models 36 days after implantation. $\mathrm{P}<0.05$ was considered to indicate a statistically significant difference.

\section{Results}

Tumor formation and growth rate. Using a lentiviral system, a stable AsPC-1 line highly expressing RFP was obtained. The 
high expression of RFP in AsPC-1 cells was confirmed using western blotting (Fig. 1).

Thirty-six days after implantation, the tumor formation rate was $100 \%(20 / 20)$ for the mice that received orthotopic tumor mass implantation, $100 \%$ (20/20) for those that received orthotopic Matrigel-tumor cell block implantation and 55\% (11/20) for the mice that received subcutaneous injection of AsPC-1 cells (Fig. 2 and Table I). All tumor-bearing mice exhibited gradual weight loss and reduced activity. Thirty-six days after implantation, the growth rates among the three xenograft models were significantly different (Fig. 3). The tumors of the mice that received the orthotopic tumor mass implantation exhibited the highest growth rate, followed by the tumors of those that received orthotopic Matrigel tumor block implantation and the tumors of the subcutaneous xenograft mice $(\mathrm{P}<0.01)$ (Fig. 3). The average tumor volume of the mice that received orthotopic tumor mass implantation was approximately $\sim 3$ fold that of the mice that received orthotopic Matrigel block transplantation and $\sim 7.4$ fold that of the mice that received the subcutaneous injection of tumor cells (Table I).

Tumor metastasis. Using an in vivo imaging system, the local invasion and metastasis of the tumors were observed in the orthotopic tumor mass implantation and Matrigel block implantation models, but not in the subcutaneous xenograft mice (Fig. 2). At day 36, the tumor-bearing mice were sacrificed and examined for metastatic tumors. In the orthotopic tumor mass xenograft model, $80 \%$ of the mice exhibited tumor metastasis, with the majority exhibiting peritoneal metastasis. Similarly, in the orthotopic Matrigel block xenograft model, $80 \%$ of the mice exhibited tumor metastasis, but the metastatic sites were slightly different. No tumor metastasis was identified in the subcutaneous xenograft model (Table I).

Anatomical and histological examinations. In the subcutaneous xenograft model, the tumors were found to adhere to the skin tightly and the tumor sections had a gray, fish-like appearance. No distant metastases were found in the thoracic or abdominal cavities. In the orthotopic tumor mass implantation model, the tumors in the pancreas were irregularly shaped and were found to adhere to surrounding tissues, such as the stomach, spleen and intestine. Metastases to the liver and peritoneum were observed (Table I). The tumors were generally rich in blood vessels on the surface and had necrosis at the center. In the orthotopic Matrigel tumor block implantation model, ascites were observed in the abdominal cavities of the mice, and metastases to the liver, peritoneum and spleen were found.

Histological examination was performed for all tumors isolated from the tumor-bearing mice. Various cell morphologies were observed, with most cells having a polygonal or spindle shape (Fig. 4). The tumors were surrounded by a fibrous stroma. No glandular differentiated cells were observed. Pathological mitotic figures were found in the tumors, which were consistent with the poorly differentiated adenocarcinoma (Fig. 4).

\section{Discussion}

Animal models are indispensable in the study of biological mechanisms and the development of therapeutics for human 

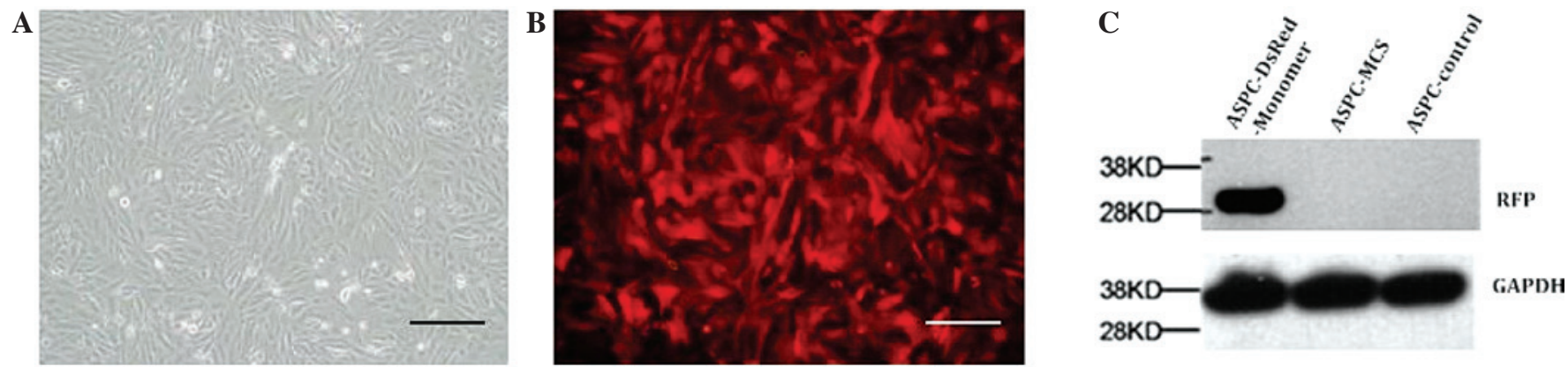

Figure 1. AsPC-1 pancreatic cancer cells were stably transfected with RFP. (A and B) Following selection, the cells that stably expressed high levels of RFP were used for the following experiments (x100). (C) The expression of RFP in AsPC-1 cells was confirmed using western blotting. RFP, red fluorescent protein.

A

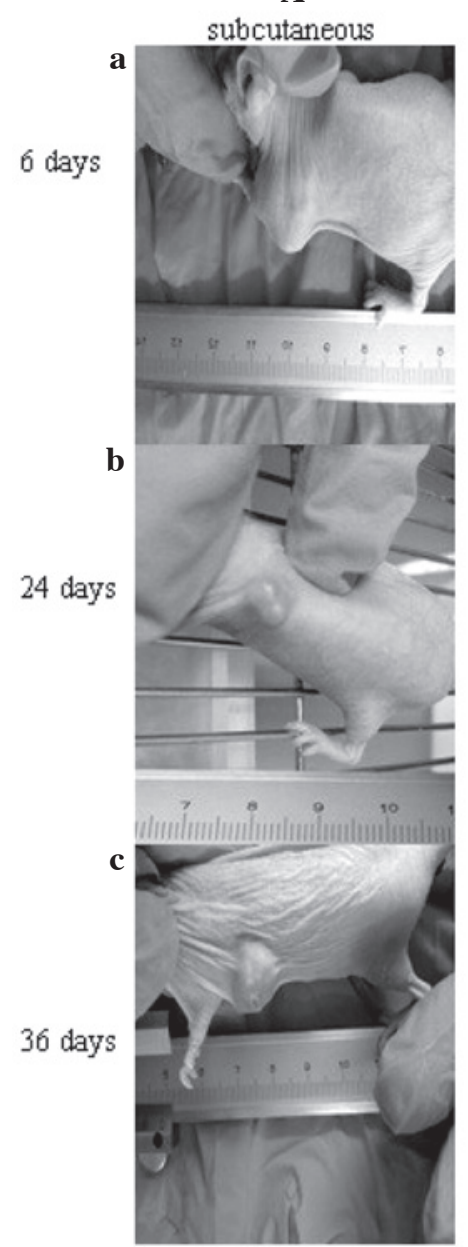

B

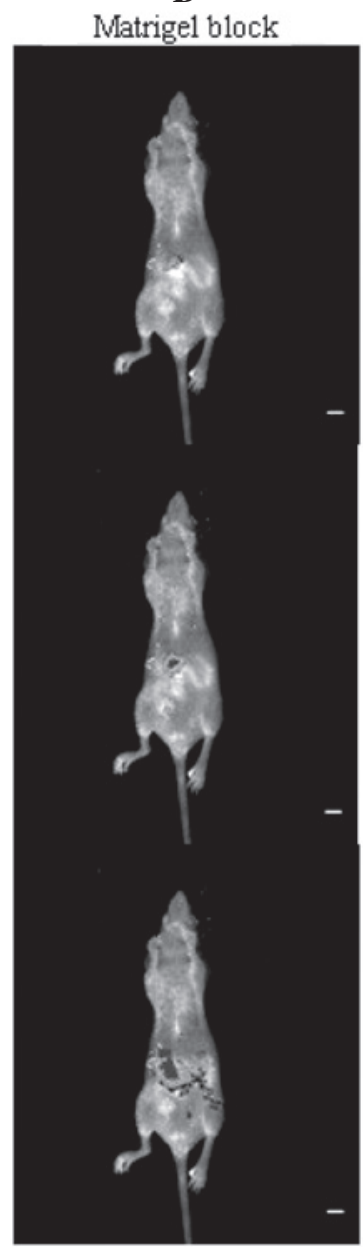

C

tumor mass

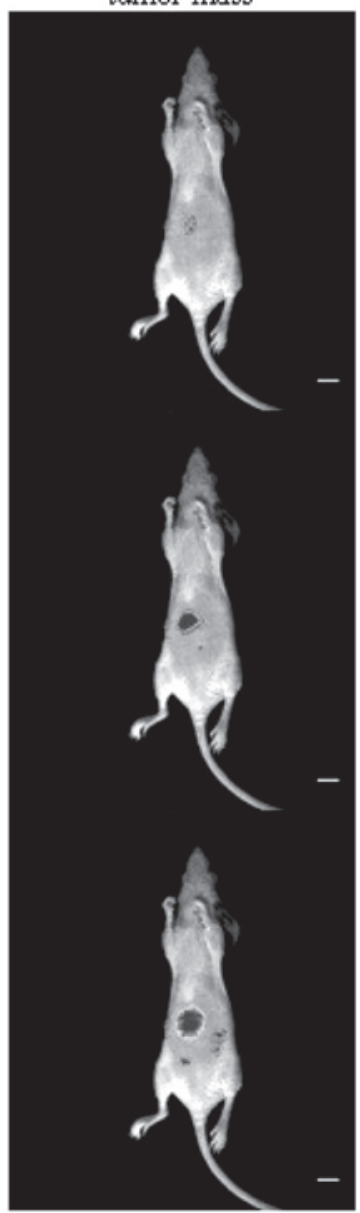

Figure 2. Tumor growth and metastasis at various time-points in each xenograft model. (Aa-Ac) The subcutaneous xenograft model was prepared by injecting RFP-labeled AsPC-1 cells into the flank of nude mice; $(\mathrm{Ba}-\mathrm{Bc})$ the orthotopic Matrigel ${ }^{\mathrm{TM}}$ tumor block xenograft model was prepared by implanting solidified Matrigel containing RFP-labeled AsPC-1 cells into the pancreas of nude mice and (Ca-Cc) the orthotopic tumor mass xenograft model was prepared by implanting a tumor block into the pancreas of nude mice. Representative images at days 6, 24, and 36 are shown. The tumor growth and metastasis in the orthotopic Matrigel tumor block and orthotopic tumor mass xenograft models were monitored using an in vivo imaging system. RFP, red fluorescent protein.

diseases. A number of mouse models of human pancreatic cancer have been reported, including the injection of pancreatic tumor cells subcutaneously or into the pancreas of the mouse $(5,6)$. In the present study, two orthotopic xenograft models were developed, in which either a tumor mass or Matrigel-tumor cell mixture was directly implanted into the pancreas of mice. The findings showed that the orthotopic tumor mass implantation model had superior performance results than the other models in terms of tumor volume and metastasis.

Due to the easy protocol of the subcutaneous xenograft model, it has been used extensively in cancer research $(11,12)$; however, the subcutaneous injection of tumor cells often results in local growth but rarely distant metastasis, and the 

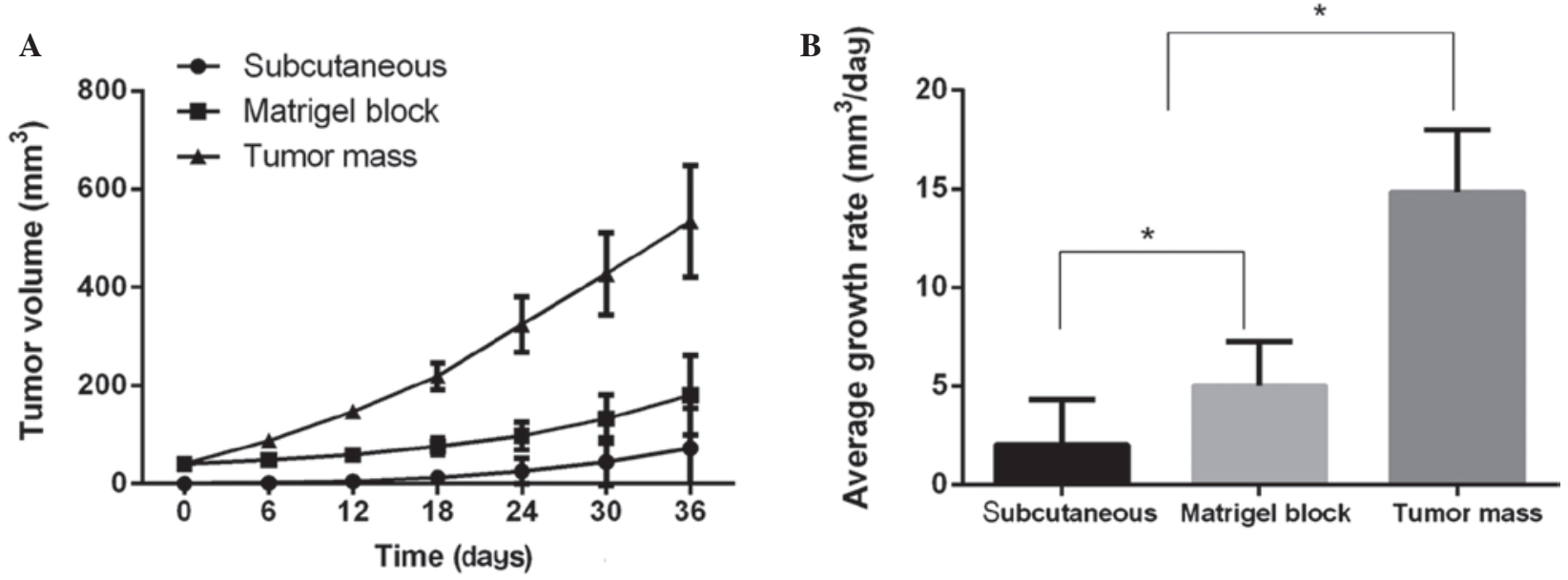

Figure 3. Tumor (A) volume and (B) growth rates in the three xenograft pancreatic tumor models. The tumors in the orthotopic tumor mass and orthotopic Matrige $^{\mathrm{TM}}$ block implantation models grew significantly faster than those in the subcutaneous xenograft model. ${ }^{*} \mathrm{P}<0.01$.

A

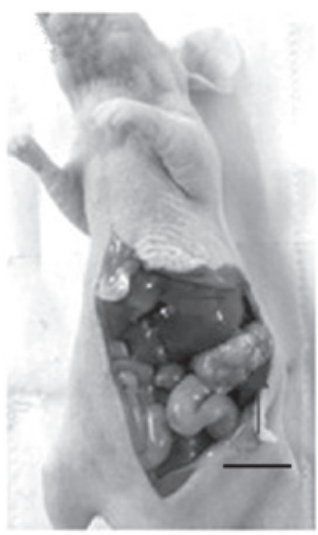

B

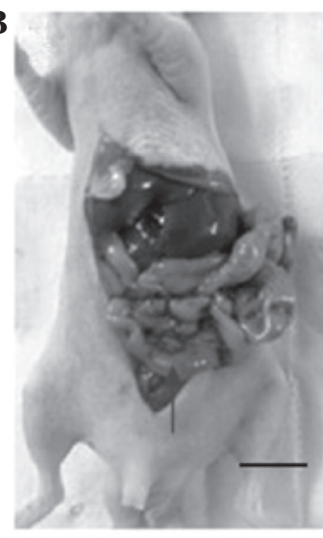

C

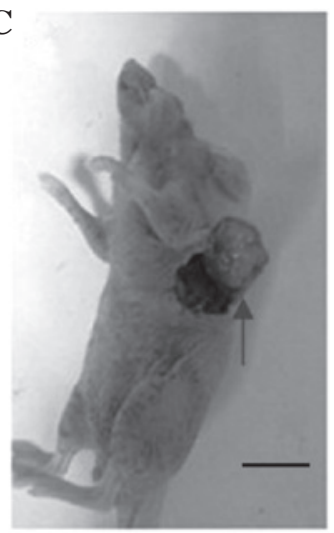

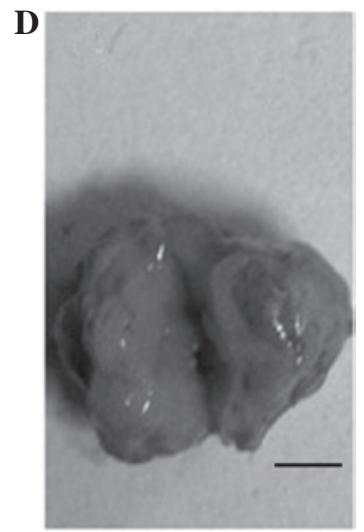

$\mathbf{E}$

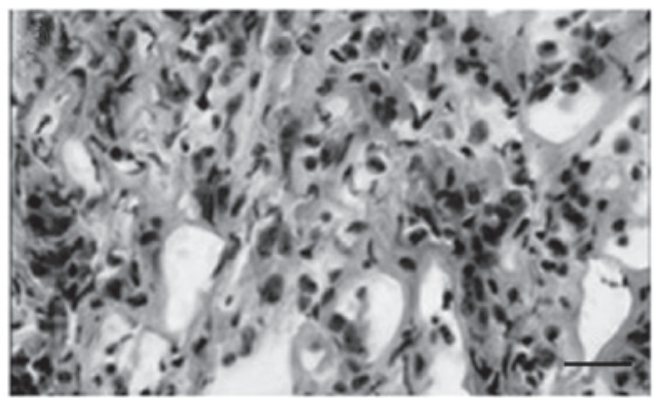

$\mathbf{F}$

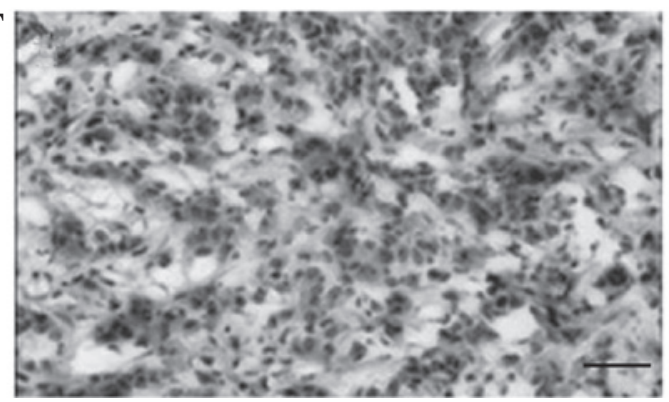

Figure 4. Anatomical and histological examinations of the tumors in the three xenograft pancreatic tumor models. (A) Orthotopic tumors and metastatic liver nodule 36 days after implantation. (B) Mesenteric metastatic nodules in the orthotopic tumor mass xenograft model. (C) Subcutaneous tumor in the subcutaneous xenograft model. (D) The orthotopic pancreatic tumor showed a gray-white, fish-like appearance. (E) HE staining of the section of the metastatic liver nodule showed characteristic tissue- and cell-atypia in histology (magnification, $\mathrm{x} 400$ ). (F) HE staining of the section of the orthotopic pancreatic tumor revealed the morphology of a poorly differentiated pancreatic adenocarcinoma (magnification, x200). HE, hematoxylin and eosin.

tumor may even fail to develop. In addition, the growth rate of a subcutaneous tumor is influenced by numerous factors, such as cell type and number. Furthermore, in light of the importance of the tumor environment to the growth and progression of tumors (13), the lack of an orthotopic environment makes the subcutaneous xenograft model less attractive for cancer research.

With regard to the orthotopic xenograft model for pancreatic cancer, the most commonly used method includes injecting a tumor cell suspension into the pancreas $(5,14)$. The orthotopic injection method was attempted in the present study; however, the results showed that there was a high risk of cell leakage, which reduced the rate of tumor inoculation orthotopically but increased the rate of peritoneal inoculation. In order to avoid this leakage associated with the orthotopic injection model, the Matrigel-tumor cell xenograft model was established. In this model, the tumor cells were first mixed with Matrigel, and the solidified block was then implanted into the pancreas. This model was $100 \%$ successful and had a $0 \%$ mortality rate under well-controlled anesthesia and with skilled surgical techniques. Metastasis is one the most prominent pathological features of pancreatic cancer. A successful animal model of 
pancreatic cancer should be able to develop metastasis. In the present study, the two orthotopic xenograft models successfully developed tumor metastasis. This property makes these two models particularly useful in the study of tumor metastasis mechanisms and intervention development.

In the models of the present study, an in vivo imaging system was used to monitor tumor growth in a real-time and non-invasive fashion. This proved very convenient for evaluating the efficiency of the models and would be beneficial in the monitoring of anti-cancer drug efficacy. The current method involved the use of RFP, which may not have been the most suitable, since its fluorescent intensity could be subject to interference by layers of biological tissues, thus leading to a limitation of the depth of fluorescence imaging. An infrared fluorescent protein with a longer wavelength or a fluorescence imaging system with higher photosensitivity would help overcome these challenges. In addition, since Matrigel is a preparation of basement membrane, its quality could vary, which would lead to numerous variations in the xenograft model. The use of a new material that could substitute Matrigel has been reported in a prostate cancer xenograft model (15). Testing the material in the pancreatic cancer xenograft model could prove beneficial.

In conclusion, two orthotopic xenograft mouse models for human pancreatic cancer were successfully developed, which could be useful in the study of tumorigenesis, tumor progression and metastasis.

\section{Acknowledgements}

This study was supported by the Scientific Innovation Team Project of Ningbo (grant no. 2013B82010), the Key Project for Social Development of Ningbo (grant no. 2011C51005) and the K.C. Wong Magna Fund in Ningbo University (Ningbo, China).

\section{References}

1. Siegel R, Naishadham D and Jemal A: Cancer statistics 2012. CA Cancer J Clin 62: 10-29, 2012.

2. Brennan MF: Adjuvant therapy following resection for pancreatic adenocarcinoma. Surg Oncol Clin N Am 13: 555-566, 2004.

3. Stojadinovic A, Hoos A, Brennan MF and Conlon KC: Randomized clinical trials in pancreatic cancer. Surg Oncol Clin N Am 11: 207-229, 2002.
4. Wolfgang CL, Herman JM, Laheru DA, Klein AP, Erdek MA, Fishman EK and Hruban RH: Recent progress in pancreatic cancer. CA Cancer J Clin 63: 318-348, 2013.

5. Yamamura K, Kasuya H, Sahin TT, Tan G, Hotta Y, Tsurumaru N, Fukuda S, Kanda M, Kobayashi D, Tanaka C, et al: Combination treatment of human pancreatic cancer xenograft models with the epidermal growth factor receptor tyrosine kinase inhibitor erlotinib and oncolytic herpes simplex virus HF10. Ann Surg Oncol 21: 691-698, 2014.

6. Nikfarjam M, Yeo D, He H, Baldwin G, Fifis T, Costa P, Tan B, Yang E, Wen S and Christophi C: Comparison of two syngeneic orthotopic murine models of pancreatic adenocarcinoma. J Invest Surg 26: 352-359, 2013

7. Troiani T, Schettino C, Martinelli E, Morgillo F, Tortora G and Ciardiello F: The use of xenograft models for the selection of cancer treatments with the EGFR as an example. Crit Rev Oncol Hematol 65: 200-211, 2008.

8. Bimonte S, Barbieri A, Palma G, Luciano A, Rea D and Arra C: Curcumin inhibits tumor growth and angiogenesis in an orthotopic mouse model of human pancreatic cancer. BioMed Res Int 2013: 810423, 2013.

9. Nangami GN, Watson K, Parker Johnson K, Okereke KO, Sakwe A, Thompson P, Frimpong N and Ochieng J: Fetuin-A ( $\alpha 2 \mathrm{HS}$-glycoprotein) is a serum chemo-attractant that also promotes invasion of tumor cells through Matrigel. Biochem Biophys Res Commun 438: 660-665, 2013.

10. Büchler P, Reber HA, Roth MM, Shiroishi M, Friess H and Hines OJ: Target therapy using a small molecule inhibitor against angiogenic receptors in pancreatic cancer. Neoplasia 9: 119-127, 2007.

11. Hakkarainen T, Särkioja M, Lehenkari P, Miettinen S, Ylikomi T, Suuronen R, Desmond RA, Kanerva A and Hemminki A: Human mesenchymal stem cells lack tumor tropism but enhance the antitumor activity of oncolytic adenoviruses in orthotopic lung and breast tumors. Hum Gene Ther 18: 627-641, 2007.

12. Poplin E, Feng Y, Berlin J, Rothenberg ML, Hochster H, Mitchell E, Alberts S, O'Dwyer P, Haller D, Catalano P: Phase III, randomized study of gemcitabine and oxaliplatin versus gemcitabine (fixed-dose rate infusion) compared with gemcitabine (30-minute infusion) in patients with pancreatic carcinoma E6201: A trial of the Eastern Cooperative Oncology Group. J Clin Oncol 27: 3778-3785, 2009.

13. McAllister SS and Weinberg RA: Tumor-host interactions: A far-reaching relationship. J Clin Oncol 28: 4022-4028, 2010.

14. Colucci G, Labianca R, Di Costanzo F, Gebbia V, Cartenì G, Massidda B, Dapretto E, Manzione L,Piazza E, Sannicolò M, et al; Gruppo Oncologico Italia Meridionale (GOIM); Gruppo Italiano per lo Studio dei Carcinomi dell'Apparato Digerente (GISCAD); Gruppo Oncologico Italiano di Ricerca Clinica (GOIRC): Randomized phase III trial of gemcitabine plus cisplatin compared with single-agent gemcitabine as first-line treatment of patients with advanced pancreatic cancer: The GIP-1 study. J Clin Oncol 28: 1645-1651, 2010.

15. Cui L, Chen P, Tan Z, Li W and Dong Z: Hemostatic gelatin sponge is a superior matrix to matrigel for establishment of LNCaP human prostate cancer in nude mice. Prostate 72: 1669-1677, 2012. 